\title{
Pre-hospital Management of Spinal Cord Injuries
}

\author{
Barth A. Green, M.D., Frank J. Eismont, M.D. and James T. O'Heir \\ Departments of Neurological Surgery, University of Miami, School of Medicine, \\ 1501 N.W. 9th Avenue, Miami, Florida, 33136, U.S.A.
}

Spinal cord injury is a multibillion dollar annual health care problem in the United States and across the world. It is considered a catastrophic disease because of the high morbidity, mortality and economic impact associated with these paralysing injuries.

We advocate a systems approach for the comprehensive management of spinal cord injury patients based on an extensive multicentre experience which has produced data supporting this concept. The front end of this system involves prevention programmes based on the philosophy that it is more sensible to avoid an injury or disease than to have to deal with its treatment and consequences, especially when therapy is so complex as it is in the case of spinal cord injuries. This phase involves public and professional education through various media modalities as well as legislative action directed towards safety equipment, speed limits, handgun restrictions, etc. Although successful in some areas, to date these prevention programmes have not affected the overall numbers of spinal cord injuries in our country or abroad. The next phase of system care involves the front end of the medical delivery component or the prehospital management phase. Modern emergency medical services in the United States evolved as a result of the classic white paper, Accidental Death and Disabilities-The Neglected Disease of Modern Society, released by the National Academy of Sciences National Research Council Committee on Shock and the Committee on Trauma, 1966. This document drew attention to the severe deficiencies existing in emergency care and served as a catalyst for a series of events directed towards improvement in the care of trauma victims. The Emergency Medical Services Systems which evolved were comprised of 15 components identified by Congress and the Emergency Medical Services Systems Act which also defined seven critical patient groups for emphasis, one of which was Spinal Cord Injuries. In 1984, there were 92000 accidental deaths in the United States with about $50^{\circ}$ o being caused by auto accidents with an economic impact estimated to be 96.9 billion dollars in lost wages and 13.8 billion dollars in medical expenses. In spite of these overwhelming figures and the significant successes of the National Emergency Medical Service programme, federal funding was eliminated as part of the budget trimming process in the early $80 \mathrm{~s}$. However, the strong foundations established by this massive federal 
effort resulted in statewide and local programmes disbursed throughout the country which have continued to provide increasing excellence in pre-hospital care for trauma victims although unfortunately without any national organisation, co-ordination or national data base (Hall et al., 1976).

Physicians and allied health professionals dealing with spinal cord injury should realise the important role that their pre-hospital colleagues play in this health care delivery system. This is best illustrated by the fact that in the United States in the 1970s the majority of patients arrived at emergency rooms with complete lesions, meaning a total lack of motor and sensory function below their level of injury. This is in contrast to the 1980s, when the majority of spinal cord injury victims are arriving with incomplete lesions, meaning they have some sparing of motor and/or sensory function. The prognosis for complete injuries is less than a 3 to $4^{\circ}{ }_{0}$ chance of spontaneous functional recovery whereas in cases with incomplete lesions, the majority of patients experience at least some degree of neurological recovery (Ducker and Perot; Green and Eismont, 1984; Green et al., 1984; Green and Wagner, 1973; Hall et al., 1976). This dramatic change in the prognosis for neurological recovery following spinal cord injury can only be attributed to the sophistication of the Emergency Medical Services and the changing of the first responders from policemen, ambulance drivers and untrained bystanders to highly skilled paramedics, well versed in advanced life support and experienced in appropriate extrication, immobilisation, assessment, monitoring and transportation techniques. It is apparent from the dramatic change in neurological status upon arrival that many victims of spinal cord injury were paralysed or their paralysis worsened by inadvertent mishandling by uneducated but well meaning first responders. These secondary injuries can also occur from systemic insults such as hypoxia or hypotension as well as from increased spinal cord or nerve root compression associated with instability and improper or inadequate spinal immobilisation during extrication or transport.

Emergency Medical Services (EMS) are divided into three major programme components; education, communication and transportation all with well established protocols. Education programmes include the training of EMTIs who are mainly responsible for patient delivery rather than accident scene triage in severe trauma cases. They are trained in basic life support and CPR certified and are given training in proper extrication and immobilisation techniques. In most areas of the United States today, EMT IIs (paramedics) who are highly skilled in advanced life support techniques are responsible for major trauma victims such as those with spinal cord injuries. These skills include the capability of intubation, defibrillation, intravenous medication administration, and very importantly in the case of spinal cord injury, neurological assessment. These paramedics serve as the frontline troops in contemporary EMS systems (Gunn, 1982).

Very little scientific research has been utilised in developing techniques and protocols for EMS systems. For this reason there is much controversy regarding the right and wrong way to care for patients. One of the most controversial issues involves the categorisation of trauma systems. Categorisation includes three levels of trauma centres with category I being the highest level. At these institutions tertiary specialised care is provided for major trauma including 
spinal cord injuries. These centres are usually within medical school complexes with around the clock in house physician coverage in all specialities and around the clock operating room, laboratory and intensive care resources. Level II hospitals have similar resources but on a 30 minute response time basis and are not set up to provide care for certain major problems such as complicated burns and spinal cord injuries. Level III includes all other hospitals not qualified for Level I or II. The main controversy lies in the question of whether a critically injured patient, including spinal cord injury victims, should be taken to the nearest lower level trauma centre or should be transported (requiring a greater length of time), to a more highly specialised level I tertiary care centre. A recent case of a gunshot wound through the chest and the heart best illustrates this debate. Following this injury, the paramedics contacted a local hospital emergency room physician who ordered them to his facility in spite of the fact that no surgeon was available and the operating room staff was not on duty in-house and there was no cardiac bypass equipment in the operating room. Although the patient was stable at the time of the referral from the accident scene, it was the opinion of the responsible physician, that gunshot wounds to the chest do not remain stable for long and the first priority should have been life saving and stabilising measures at the nearest emergency room. The patient was taken to the local hospital and the operating team with a thoracic surgeon valiantly tried to save this man's life but he died on the operating room table resulting in a multimillion dollar law suit. The plaintiff's attorney claims that if the patient was allowed to bypass the local hospital 5 minutes from the accident scene, and was taken to the tertiary care centre 20 minutes further, the patient would have survived with the appropriate team and equipment. The defence attorney claimed that a person suffering a gunshot wound through the heart are known to become rapidly unstable and it was appropriate to take the patient to the nearest facility for stabilisation. Additionally they contend that it is well documented that gunshot wounds through the heart are fatal injuries and that the ultimate outcome would not have changed regardless of where the patient was transported. Each side had a large number of expert physician witnesses with equally good credentials supporting their 180 degree differing opinions. Ultimately, a jury of lay persons, without any knowledge of emergency medical services or trauma care, will set a legal precedent which will decide the direction of patient triage in future years. It is our contention that patients suffering a spinal cord injury, who are physiologically stable with regards to vital signs, be transported past the local hospital on to the tertiary care centre if the transport time is reasonable, i.e., less than 1 hour. If such a facility is not available within this time constraint, then it is appropriate for the patient to be taken to the nearest trauma centre for stabilisation and for secondary transport to be initiated as quickly as possible into the tertiary facility. This opinion is also controversial.

Another area of controversy involves the use of nasogastric tubes and Foley catheters. Paramedics are allowed to intubate, defibrillate, and administer I.V. medications, etc., but are not allowed to insert a Foley catheter or nasogastric tube which are essential components of an emergency room triage. Another major controversy involves patient extrication, immobilisation and transportation. It is generally agreed upon that the patient should not be left at the accident 
scene in a position in which they are found, but should be moved into a neutral position and immobilised for transport. Just how to move and manage these patients is quite controversial. These issues will be dealt with in the following protocols for accident scene management. However, they only represent the opinion of these authors and do not necessarily represent the 'acceptable norm' in this country or abroad (Hall et al., 1976; Young, 1978).

\section{Accident scene management}

At the scene of an accident, the initial consideration is recognition of the patient with a spinal cord injury. The following list includes the most significant signs and symptoms found in patients with acute spinal cord injuries.

1. Motor signs: weakness or paralysis of extremities and/or trunk muscles.

2. Sensory signs: absence or alteration of sensation of trunk and/or extremities.

3. Incontinence: loss of control of bladder and/or bowels.

4. Superficial signs: abrasions, lacerations or deformities of the spine, neck or head regions.

5. Pain: tenderness or pain on palpation of the spine or neck. The patient's neck or back should not be moved to determine if it is painful, it should only be palpated.

In addition to the signs and symptoms, one must be aware of the fact that any unconscious patient must be considered to have a spinal cord or spinal column injury until proven otherwise. Also, an injury to other systems (e.g. head injury) may mask a spinal cord injury; conversely, a spinal cord injury can mask other system injuries (e.g. visceral rupture or fracture of long bones).

Once the accident victim has been identified as a possible spinal column or spinal cord injury, the following priorities should be followed.

\section{Respiratory}

\section{Placement of an appropriate and secure airway}

In an awake patient, to prevent gagging, use a bite stick, i.e. a cut-off standard oral airway or a tongue blade wrapped in tape. In an unconscious patient, a full standard oral airway should be inserted or an EOA (esophageal obturator) utilised. The airway should always be taped to prevent loss in case of emesis or seizure or with combative patients. If emesis occurs, an infant bulb syringe with the tip cut off is of benefit for removing stomach contents or blood clots from the oral cavity although standard suction tubing is still necessary for deeper respiratory tree toilet. The neck should never be moved out of a neutral position in order to establish an airway in any patient suspected of having a spinal injury.

If the insertion of an airway does not by itself result in effective ventilation, as determined by auscultation of the lungs, then the patient should be intubated. If intubation is necessary, the use of an EOA (i.e. pharyngeal intubation) or blind nasotracheal intubation are preferable at the accident scene because of the 
comparative ease of placement without the need to hyperextend the head or neck. Such equipment is now standard in all paramedic rescue kits.

\section{Artificial assistance}

If the patient for any reason has insufficient respiratory excursions, then ambubagging should be immediately initiated. Inadequate respiration commonly occurs because of paralysis of intercostal muscles and/or diaphragm muscles, aspiration, atelectasis, or from direct trauma to the rib cage or lungs. Other signs of respiratory distress may include cyanosis or excessive retraction of accessory neck muscles associated with respiratory efforts.

\section{Proper environment}

Patients with spinal cord injuries should receive oxygen supplementation at all times during the accident scene management and transportation phases. This should be administered via nasal cannula or $\mathrm{O} 2$ mask. Often, a patient with a spinal cord injury may appear to be breathing adequately, but on admission to a hospital, baseline arterial blood gases may be far below acceptable levels. An arterial blood gas PO2 in the 60 to 70 range or a PCO2 of 60 may be acceptable in a patient with a chronic spinal cord injury, but in the acutely injured individual, one should maintain a minimum PO2 value of at least 100 and a PCO2 of less than 45 . These levels should be achieved because of the compromised blood supply and disrupted tissue metabolism which is well documented in acutely traumatised spinal cords (Ducker and Perot; Guttmann, 1976; Kahn et al.). The presence of smoke or noxious gases at the accident scene further necessitates the immediate establishment of such a controlled environment.

\section{Cardiovascular}

\section{Stop active haemorrhage}

Significant bleeding points should be treated with appropriate pressure dressings. Penetrating objects to the neck or spinal region should not be removed until the patient is in the hospital and blood transfusions and ORs are available. If objects are too long or too large to allow effective patient handling, they may be shortened to a length of several inches from the point of entry. Control of haemorrhage from spinal or neck bleeding sites should be attained by 'localised pressure' rather than by any form of circumferential dressing. A large bore intravenous line should be started on all spinal cord injury patients and Ringer's lactate solution infused.

\section{External cardiac massage when necessary}

Immediate re-establishment of circulation to neural structures is of critical importance in any effort directed toward salvage of neurological function. In certain cases, defibrillation is necessary as determined by the EKG tracing obtained by the paramedic with ongoing consultation with the ER physician. 
Various cardiogenic drugs may be administered when indicated, depending upon the EKG and other cardiovascular parameters.

\section{Treat shock}

Spinal cord injured patients may present with neurogenic shock, haemorrhagic shock or a combination of both. In most cases one or the other type is clinically predominant. In either case there is a low blood pressure and a relatively low body temperature. The key to the differential diagnosis is the bradycardia (usually a pulse below 60) observed in neurogenic shock and the relative tachycardia (usually a pulse above 100) usually seen in haemorrhagic shock. The nature of the pulse is usually slow and regular in neurogenic shock, in contrast to the rapid irregular pulse noted in cases of haemorrhagic shock. Neurogenic shock is associated with a loss of sympathetic nervous system control of the peripheral vascular tone of the extremities resulting in pooling of blood and inadequate central blood return to maintain sufficient cardiovascular function. The bradycardia is due to the loss of sympathetic inhibition of the parasympathetic effects of the vagus nerve. Patients in neurogenic shock usually present with a systolic blood pressure of approximately $70 \mathrm{~mm} / \mathrm{Hg}$ or lower and a pulse below 60 . The quickest and most effective treatment for either type of shock is to place the patient in a Trendelenberg position of 30 to 40 degrees which immediately decreases the lower extremity pooling of blood and increases central return of blood. The effect of the Trendelenberg position may be complimented by use of a MAST suit to collapse the lower extremity and abdominal vasculature. These suits must be used with caution in cases of suspected lumbar or thoracolumbar junction fractures, because they may aggravate the neurological injury especially in patients with severe spinal column disruption and instability. Shock suits are not only being carried in ambulances but are also being used in many ICUs across the country. The more specific treatment for haemorrhagic shock is fluid replacement utilising blood and volume expanders.

The specific treatment for neurogenic shock at the accident scene, after decreasing peripheral pooling by positioning and/or the use of a shock suit, should include the administration of $0.4 \mathrm{mg}$ of atropine intravenously to help block the dominant vagal effect (i.e. bradycardia). This is only a temporary measure, but most often will result in an increase in pulse rate, resulting in increased cardiac output during the brief period it takes a patient to arrive at the hospital where cardiac index parameters can be monitored with the use of a Swan-Ganz catheter. The overriding consideration must be to maintain perfusion of vital organs including the spinal cord during the early minutes and hours following injury. Caution must be used in delivering high volumes of fluids to patients in neurogenic shock in contrast to patients in hypovolemic or haemorrhagic shock. In neurogenic shock there is usually sufficient blood volume, but the problem exists in the distribution of fluids. Large increases in volume may cause the patient to develop cardiogenic failure, i.e. trading off one problem for another. 


\section{Splinting the patient}

Regardless of the posture in which the spinal cord injury victim is found following the accident, all patients should be placed in a neutral supine position and splinted from the top of the head to the bottom of the buttocks. This can often be accomplished simultaneously with assessment and stabilisation of respiratory and cardiovascular parameters by accident scene personnel working together on all priorities. It has been estimated that as many as $20^{\circ}{ }_{0}$ of spinal injuries involve multiple levels which is why the entire spinal column must be immobilised (Green and Eismont, 1984; Green et al., 1984; Hall et al., 1976). The best method of splinting the patient is using a rigid straight board which can either be made of plywood or a synthetic material. It can be combined with either towel rolls, sand bags, or a specially designed orthosis on either side of the head and neck and wedged in against the shoulders. Tape or velco straps should be placed snuggly over the forehead and more loosely over the chest so that respiratory excursions are not impeded and firmly over the pelvis, knees and ankles (Hall et al., 1976). Special consideration must be given to patients with fixed anatomical variations such as kyphoscoliosis or ankylosing spondylitis. Patients should never be forced into a position if such manoeuvres are associated with pain or obvious deformity (Hall et al., 1976).

\section{Extrication}

All patients should be placed in a neutral position. When moving a patient to a neutral position, the head must not be maintained in flexion, extension or rotation. With very gentle traction with hands locked under the jaw and neck, the patient's head and neck should be placed in alignment with the axis of the body. As long as the neutral position is not surpassed, no further damage to the spinal cord will occur with this manoeuvre. Traction with weights should never be applied at the accident scene as there is the danger of overdistraction in cases of severe spinal column disruption which could result in secondary injury with aggravation of neurological deficit. Some recent techniques utilise a small amount of weight (5-10 lbs.) during transportation without prior X-ray, but this protocol is not widely accepted. Chin straps are discouraged because of problems with airway access, especially in cases of emesis when a patient, with his mouth held tightly shut, has a higher risk of aspiration. Generally, the use of soft or rigid cervical collars for the immobilisation of these patients should be avoided. These devices can give the rescuers and the victim a false sense of security. If stressed, they will allow mobility of the neck. In addition, a relatively inelastic collar may act as a tourniquet to the neck which invariably responds to trauma by swelling. Such constriction may compromise blood and air flow. Collars may also shield from view a previously unidentified site of venous or arterial injury and haemorrhage, i.e. an expanding haematoma or may mask a ruptured trachea and the development of subcutaneous emphysema. In addition, the application of these devices may result in a greater deformity of the neck which may be associated with increased neurological deficit.

In moving a potential spinal cord injury victim, the most important factor is for the paramedic to use common sense. No two patients present in the same 
position or with the same extrication or immobilisation challenges. The goal of a successful extrication is to move the patient into a neutral supine position as quickly and safely as possible. We previously advocated the use of a logroll manoeuvre for this purpose. A cadaver study in our clinic and human studies have shown that such a manoeuvre does not offer any significant degree of stability. The use of a four man lift is equally inadequate. Since this study, we suggest the use of scoop stretchers to be placed under patients who are difficult to move, in order to transfer them without lifting to an appropriate splint i.e. spine board or rigid stretcher. However, scoop stretchers are open down the centre where the spinal column rests and requires the most support. For that reason, a scoop stretcher alone should only be used to move the patient quickly onto a spine board. Our cadaver study showed that the combination of the scoop stretcher and the spine board provides adequate splinting and support for the spinal column and may prevent secondary injuries from occurring with the more conventional logroll or lift manoeuvres. The scoop stretcher mounted on the spine board also affords additional side to side limitation of movement because of the slope of the side panels.

\section{Transportation}

In order to minimise the chances of aspiration or shock, regardless of etiology, we recommend that all spinal cord injury patients (or head injury patients with possible associated spinal cord injuries) be transported splinted supine in a Trendelenberg (head down) position of 30 to 40 degrees of tilt. The more extreme tilt should be used only if the systolic blood pressure drops below 70 $\mathrm{mm} / \mathrm{Hg}$. If a patient vomits while at this angle, the emesis will more likely follow gravity and come out of the nose and mouth. In cases of interhospital transfer of spinal cord injury patients, a nasogastric (sump) tube should be inserted and the stomach contents emptied, and during transportation the nasogastric tubing should be left open to gravity drainage or be connected to suction. These steps can prevent the dire consequences of aspiration in spinal cord injury victims who very often have associated respiratory system compromise. It must be noted that there is a significant incidence of vomiting in patients transported by ambulance and an even higher incidence in patients transported by either helicopter or fixed wing aircraft.

It is imperative that a specially trained paramedic attend each patient at the accident scene and in transit to the hospital to insure that all priority measures are employed. Although speed of extrication and transportation are important factors, they must not be placed as a priority above the principles discussed in the preceding paragraphs. The emphasis must be placed on getting a viable patient to the Emergency Room with all systems stabilised in order to enhance the potential for maximum neurological recovery. The choice of ambulance, helicopter or fixed wing transportation must depend upon availability of equipment, distance to be covered, geographical location, time of day, traffic patterns, weather and the general physical and neurological status of the patient. Often, a combination of these vehicles in a well co-ordinated effort provides an optimal emergency evacuation and transportation effort. It is almost impossible to monitor vital signs in an ambulance and even more difficult with the noise and 
vibrations of a helicopter or fixed wing aircraft. The recent development of small portable Doppler stethoscopes makes the monitoring of both the blood pressure and pulse more feasible during transportation.

Also of importance in the pre-hospital phase is a sophisticated communications system allowing consultation between the accident scene rescue team and the physician at the nearest trauma centre and/or spinal cord injury centre. Communications between accident scene personnel and inhospital team members allows the mobilisation of appropriate ER equipment and staff, as well as radiological, surgical and intensive care facilities required for optimal diagnostic and therapeutic regimens. In certain cases, it is desirable to transport the patient to the nearest designated ER, where appropriate first aid, diagnosis and further stabilisation can be accomplished. Then, when appropriate, a timely secondary transfer to a spinal cord injury centre should be initiated. However, if a spinal cord injury centre is readily accessible, the patient should be transported directly to the centre from the accident scene. Transfer from emergency rooms or hospitals into the spinal cord injury centre should always be preceeded by a physician to physician telephone conference and should be co-ordinated with the EMS system. In these cases, patients should always be accompanied by all $\mathrm{ER}$ and hospital records including laboratory tests and X-rays to expedite triage and treatment in the spinal cord injury centre.

Presently, across the United States and around the world, protocols are being implemented assessing new drugs for administration to spinal cord injury victims within the first minutes and hours following their accident. In the United States, two neuropeptides Naloxone (Narcan) and Thyrotropin Releasing Hormone (TRH) are being assessed in multicentre studies with regards to their effectiveness in limiting and reversing the neurological deficit associated with these devastating injuries. Some of these protocols include the first dose administration at the accident scene within minutes following the traumatic event. If any of these medications prove effective in these trials, it would be anticipated that their administration by first responders would be extended throughout this country and abroad. Another area of rapidly evolving technology involves the development and testing of new equipment for extrication, stabilisation and reduction of spine fractures to be utilised in the prehospital phase. More sophisticated monitoring devices and treatment protocols are also becoming available on a rapidly increasing basis. At the same time as spinal cord injury specific treatments and equipment are becoming more sophisticated, so is the management of the multisystem injuries which are associated with the majority of spinal cord injury cases. Just as in the case of head injury, there is no doubt that shock and hypoxia play a major role in the pathophysiology of these paralysing lesions and their early recognition and effective treatment can only improve the outcome of paralysis victims. We strongly encourage the development of multicentre and multinational prospective, randomised and well controlled studies to further develop and evaluate treatment protocols for acute spinal cord injury. We believe that only through such wide spread co-operation can significant gains be made in reducing the morbidity, mortality and cost of these devastating injuries. 


\section{References}

Ducker TB, Perot PL National Spinal Cord Injury Registry. Div. of Neurosurgery M.U. of S. Car. 80 Barre St., Charleston, SC 21401

GreEN BA, Eismont, FJ 1984 Acute spinal cord injury: a systems approach. Central Nervous System Trauma Vol. 1, No. 2

GreEN BA, KLOSE KJ, GOLDBERg ML 1984 Clinical and research considerations in spinal cord injury. In: Central Nervous System Trauma Status Report, Chapter 22

GreEN BA, KLOSE KJ, O'HEIR J 1982 Emergency medical services and the pre-hospital care of spinal cord injury. In: Green, Marshall and Gallagher (eds) Intensive Care for Neurological Trauma and Diseases, Chapter 1

GREEN BA, WAGNER FC 1973 Evolution of edema in the acutely injured spinal cord: a fluorescent microscopic study. Surgery and Neurology 1:98

GuNN JS 1982 Spinal cord injury statistics. Experience of the Regional Spinal Cord Injury Systems. Phoenix, Arizona

Guttmann Sir Ludwig 1976 Spinal Cord Injuries-Comprehensive Management and Research. Blackwell Scientific Publications, Oxford London, Edinburgh and Melbourne, 2nd edn

Hall WJ, Green BA, Colodonato JP 1976 Spinal cord injury: emergency management. Emergency Medical Services 5: May-June

KAHN T, GREEN BA, RAIMONDI AJ Energy metabolism of the acutely injured

YouNG JS 1978 Initial hospitalization and rehabilitation costs of spinal cord injury. In: The Orthopedics Clinics of North America. W. B. Saunders Co., Phil., London, Ontario, Vol. 9 (2) 